\title{
Estudo retrospectivo de lesões inflamatórias periapicais crônicas diagnosticadas na região de Barretos entre 2004 a 2014 com avaliação de concordância entre diagnóstico clínico/histopatológico.
}

\author{
A retrospective study of chronic periapical inflammatory injury \\ diagnosed in Barretos region from 2004 to 2014 evaluated by \\ agreement between clinical / histopathologic diagnosis. \\ Luana Gabriela Alves ${ }^{1}$, Felipe Camargo de Freitas ${ }^{2}$, Alex dos Reis Inhota ${ }^{3}$, Renata Hebling Marins ${ }^{4}$, \\ Raphael Carlos Comelli Lia* \\ 1, 2, 3 Centro Universitário da Fundação educacional de Barretos - UNIFEB \\ ${ }^{4}$ Universidade de São Paulo - UNAERP \\ * Campus de Araraquara Universidade Estadual Paulista Júlio de Mesquita Filho, Faculdade de Odontologia.
}

\begin{abstract}
Resumo
Patologias inflamatórias periapicais crônicas são universais, abrangem o ápice e são entidades de transição. O objetivo é comparar as diferenças entre os diagnósticos clínicos, radiográficos e histopatológicos, para demonstrar a importância da acuracidade diagnóstica. Foram analisados de forma clínico/radiográfico/ histopatológica 449 casos, levantados, de lesões periapicais inflamatórias crônicas; a Periodontite Apical Crônica (PAC) com suas variáveis, representou 258 (57\%) dos casos; sendo, 215 deles processos crônicos ativos, 26 crônicos em evolução colagênica, 16 crônicos ativos em abscedição e 1 crônico epiteliado, já para os Processos Císticos Epiteliados Odontogênicos Inflamatórios Radiculares Periapicais (CEOIRP), foram encontrados $191(43 \%)$ casos. Referente às faixas etárias, tanto a PAC quanto o CEOIRP predominaram na quinta década de vida e no gênero feminino. De 283 diagnósticos clínicos como PAC, 104 (36\%) dos casos não foram confirmados por exame histopatológico, sendo caracterizados como "CEOIRP". De 132 diagnósticos clínicos de CEOIRP, 59 (44\%) não foram confirmados pelo histopatológico, sendo caracterizados como PAC. De 34 casos não definidos clinicamente, 27 deles tiveram diagnóstico histopatológico confirmado como periodontite apical crônica PAC e 7 deles como Cisto inflamatório radicular CEOIRP. Concluiuse que nessas condições o diagnóstico histopatológico não confirma a hipótese diagnóstica definida por achados clínicos e radiográficos.
\end{abstract}

Palavras chave: Periodontite apical crônica; Granuloma periapical; cisto radicular; divergências de diagnóstico clínico e histopatológico.

\footnotetext{
Abstract

Autor para correspondência: Luana Gabriela Alves

E-mail: luana.alves.odonto@gmail.com

Telefone: (16) 99124-3660

Recebido em: 18/10/2016

Aceito para publicação em: 23/07/2017
}

Periapical chronic inflammatory diseases are universal, covering the summit and are transitional entities. This study aims to compare the differences between the clinical diagnosis, radiographic and histopathologic diagnosis to demonstrate the importance of diagnostic accuracy. Clinical forms were analyzed, radiographical,

https://doi.org/10.4322/1980-0029.092016 
andhistopathological examinations were also carried out. 449 cases were collected, with chronic inflammatory periapical lesions; and Chronic apical periodontitis (PAC) with its variables, represented $258(57 \%)$ of the cases; being 215 of them active chronic diseases, 26 chronic evolving collagen, 16 chronic active in abcess and 1 chronic epithelized, as for cystic processes epithelized Odontogenic Inflammatory Root Periapical (CEOIRP), it was found 191 (43\%) cases. By age groups, both the PAC and the CEOIRP predominated in the fifth decade of life and females. 283 clinical diagnoses such as PAC, 104 (36\%) cases were not confirmed by histopathology being characterized as CEOIRP, 132 clinical diagnostic CEOIRP, 59 (44\%) were not confirmed by histopathological being characterized as PAC. 34 cases not defined clinically, 27 of them had histopathological diagnosis confirmed as chronic apical periodontitis PAC, and their 7 as root inflammatory cyst CEOIRP. It was concluded that under these conditions the histopathologic diagnosis does not confirm the diagnosis defined by clinical and radiographic findings.

Keywords: chronic apical periodontitis; periapical granuloma; radicular cyst; clinical diagnosis and histopathological differences.

\section{Introdução}

As causas de patologias inflamatórias periapicais são variáveis, incluindo as respostas imunogênicas e idiopáticas, bem como reações de corpo estranho. Por isso, com muita frequência, é comum observar estas três condições de reação concomitantemente agindo de uma maneira especial na evolução da inflamação regional. Tais reações dependem das condições periapicais, como a persistência da microbiota estabelecida e de modificações locais, como as representadas pela presença de cristais de colesterol e/ou materiais odontológicos (exógenos) que possam ser extravasados, indigeríveis (Lia et al., 2004), A maioria dessas reações granulomatosas periapicais e de processos císticos radiculares é encontrada ocasionalmente durante exames radiográficos de rotina (Neville et al., 2009).

\section{Características clínicas e radiográficas.}

Assim como nos processos císticos, a maioria dos granulomas periapicais é assintomática, mas pode desencadear dor ou sensibilidade se ocorrer uma exacerbação inflamatória. Por característica, o dente envolvido não apresenta mobilidade ou sensibilidade significativa à percussão. $\mathrm{O}$ tecido mole subjacente ao ápice pode ou não apresentar alguma sensibilidade. O dente não responde aos testes pulpares térmicos ou elétricos, a menos que a necrose pulpar esteja relacionada a um único canal de um dente multirradicular. Os granulomas periapicais representam quase $75 \%$ das lesões inflamatórias periapicais e $50 \%$ deles não responderam a técnicas endodônticas conservadoras (Neville et al., 2009). Em geral, os pacientes com cistos periapicais não apresentam sintomas, a menos que exista uma exacerbação inflamatória aguda, como pode ocorre em "cistos Phoenix" (Neville et al., 2004). Além disso, se o processo cístico atingir tamanho considerável, podem ser observadas tumefação e sensibilidade leve (Shafer et al., 1987). Associado à ocorrência de um aumento volumétrico, podem ocorrer mobilidade e deslocamento dos dentes adjacentes (Regezi et al., 2000). Pela perda da vitalidade pulpar, como já salientado, o dente de origem não responde aos testes pulpares térmicos e elétricos, exceto em dentes multirradiculares, que podem eventualmente manter vitalidade da polpa de canais não envolvidos no processo. O padrão radiográfico é semelhante ao do granuloma periapical (Regezi et al., 2000). A radiolucidez da periodontite apical crônica é de tamanho variável pode ser observada com a perda da lâmina dura, com fronteiras bem ou mal definidas, com ou sem condensação óssea envolvida. A radiolucidez juntamente com inserção perpendicular pode levar a um diagnóstico sugestivo de cisto. No entanto, a inserção oblíqua pode direcionar um diagnóstico de granuloma periapical, mas esses dados não são conclusivos (Neville et al.,1998). O tamanho é bem variável, ocorrendo como lesões pequenas pouco perceptíveis, até radiotransparência excedendo dois centímetros de diâmetro. A reabsorção óssea radicular não é incomum, embora as lesões maiores que do que $200 \mathrm{~mm}^{2}$ representem muitas vezes cistos periapicais, não excluindo a possibilidade de granuloma periapical, vários pesquisadores não têm sido capazes de distinguir granuloma (Neville et al., 2009) (Figuras 1 e 2).

\section{Características Histológicas}

Neville et al. (1998) afirma que granulomas periapicais consistem em tecido de granulação inflamado, contido em um invólucro de tecido 
conjuntivo fibroso. Tal tecido possui um infiltrado linfocítico variável, associado a neutrófilos, plasmócitos, histiócitos e, menos frequentes, mastócitos e eosinófilos. Já Ferreira et al. (2016) diz que granulomas periapicais apresentam um infiltrado mononuclear localizado e intenso que consiste em linfócitos e células plasmáticas difundidos num tecido conjuntivo fibroso contendo numerosos fibroblastos e vasos. Áreas focais de neutrófilos infiltrados no tecido de granulação podem ser observadas, por vezes, em casos de granulomas com exacerbação aguda. Quando há uma presença maior de plasmócitos, glóbulos

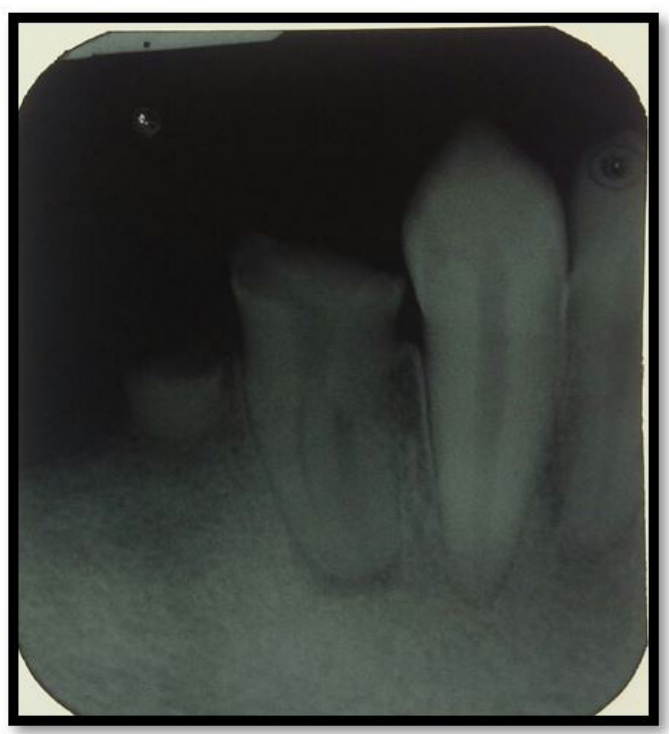

Figura 1. Periodontite apical crônica

Fonte: Centro universitário da Fundação educacional de Barretos - UNIFEB.

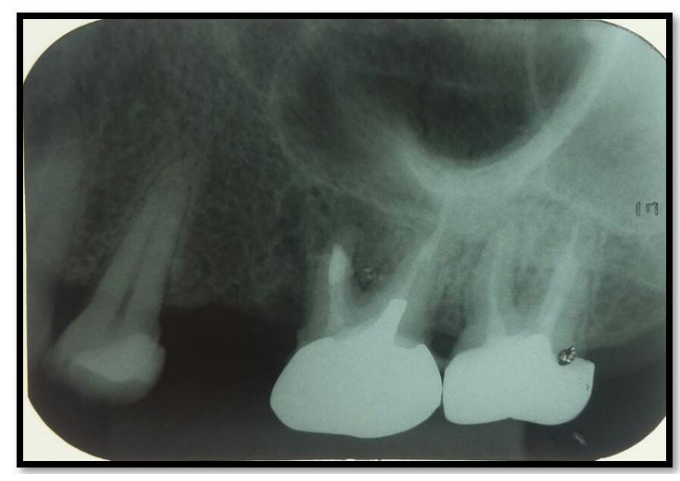

Figura 2. Cisto radicular

Fonte: Centro Universitário da Fundação de Barretos - UNIFEB. eosinófilos de gamaglobulina podem ser observados como corpúsculos de Russell. Além disso, grupos de partículas levemente basofílicas, também nos agregados plasmocitários, são detectados como corpúsculos de pironina (Neville et al., 2009) (Figura 3). Cistos radiculares é caracterizadas por uma lesão cavitária que contém líquido, as células necróticas apresentam diversos estágios de degradação e número eventual ou variado de células inflamatórias.

Nos cistos radiculares, cristais de colesterol podem estar presentes, dando ao conteúdo cístico, por intensidade, uma típica coloração amarelada, com cavidade revestida por epitélio escamoso estratificado. Raramente queratinizado, o epitélio pode apresentar hiperplasia e exocitose leucocitária (Regezi et al., 2000).

A parede cística apresenta áreas de tecido conjuntivo de frouxo a denso, com zonas de tecido de granulação e infiltrado inflamatório composto basicamente de mononucleares (Neville et al., 2009) (Figura 4).

\section{Material e método}

Levantamento bibliográfico em livros e artigos científicos com pesquisa utilizando os unitermos: periodontite apical cônica, granuloma periapical e cisto radicular. Foram levantados de prontuários clínicos com conclusão diagnóstica definida por laudos histopatológicos. Analisou-se exames clínicos, radiográficos e histopatológicos dos pacientes diagnosticados com periodontite apical crônica (PAC) ou granuloma periapical crônico

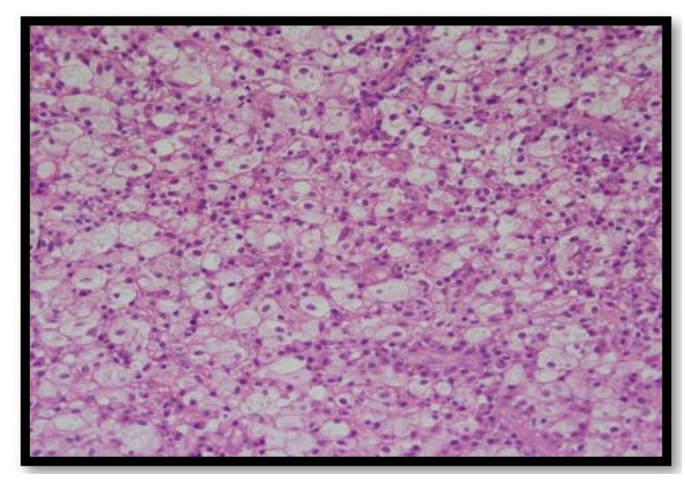

Figura 3. Corte histológico de periodontite apical crônica com predominância de um infiltrado inflamatório de prevalência de mononucleares (macrófagos espumosos, linfócitos e plasmócitos). Fonte: Serviço de Patologia do Centro Universitário da Fundação Educacional de Barretos - UNIFEB. 
e cisto radicular no período de 2004 até 2014. Com o auxílio do sistema operacional Excel 2013, estudamos comparativamente o diagnóstico clínico e os resultados histopatológicos, dispondo-os em gráficos. Todos os laudos são de pacientes atendidos na clínica odontológica do Centro Universitário da Fundação Educacional de Barretos - UNIFEB com conclusão diagnóstica definida pelo Serviço de patologia da mesma instituição.

\section{Resultados e Discussão}

A periodontite apical crônica foi a lesão levemente predominante neste estudo. $57 \%$ das lesões foram diagnosticadas como PAC, enquanto $43 \%$ como

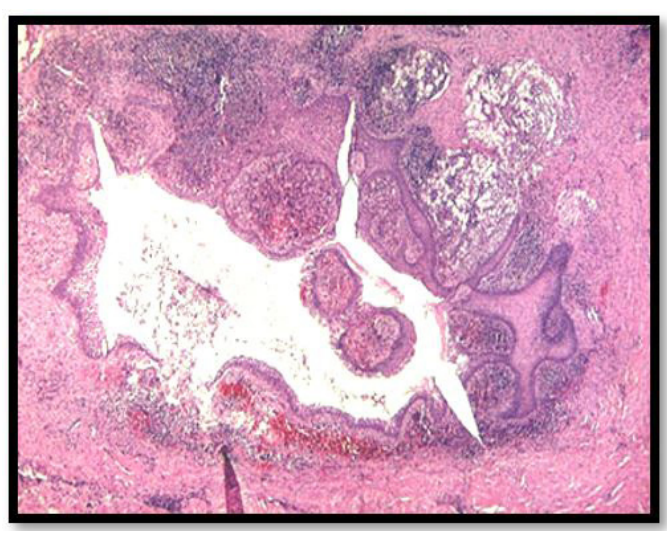

Figura 4. Corte histológico de cisto radicular revestido por epitélio escamoso estratificado.

Fonte: Serviço de Patologia do Centro Universitário da Fundação Educacional de Barretos - UNIFEB.
CEOIRP. Estes resultados são semelhantes aos relatados por Block et al. (1976), Morse et al. (1973), Baumann \& Rossman (1956), Grossman \& Ether (1963), Linenberg et al. (1964), Mortensen et al. (1970), Patterson et al. (1964), Wais (1958) e em condição inversa dos relatados por Bhaskar (1966), Lalonde \& Luebke (1968) e Priebe et al. (1954). Estas divergências com relação à incidência de periodontite apical crônica podem estar relacionadas com critérios individuais de cada investigador (Rocha et al., 1998). Foram analisados de forma clínico/radiográfico/histopatológica 449 casos levantados de lesões periapicais inflamatórias crônicas. A Periodontite Apical Crônica (PAC), com suas variáveis, representou 258 (57\%) dos casos, sendo 215 deles processos crônicos ativos, 26 crônicos em evolução colagênica, 16 crônicos ativos em abscedição e 1 crônico epiteliado. Já para os Processos Císticos Epiteliados Odontogênicos Inflamatórios Radiculares Periapicais (CEOIRP), foram encontrados 191 casos (43\%. Vide gráfico 1). Referente às faixas etárias, tanto a PAC quanto o CEOIRP predominaram na quinta década de vida e no gênero feminino (Gráficos 2 e 3 ). De 283 diagnósticos clínicos como PAC, $104(36 \%)$ dos casos não foram confirmados por exame histopatológico, sendo caracterizados como CEOIRP. De 132 diagnósticos clínicos de CEOIRP, 59 (44\%) não foram confirmados pelo histopatológico, sendo caracterizados como PAC. De 34 casos não definidos clinicamente, 27 deles tiveram diagnóstico histopatológico confirmados
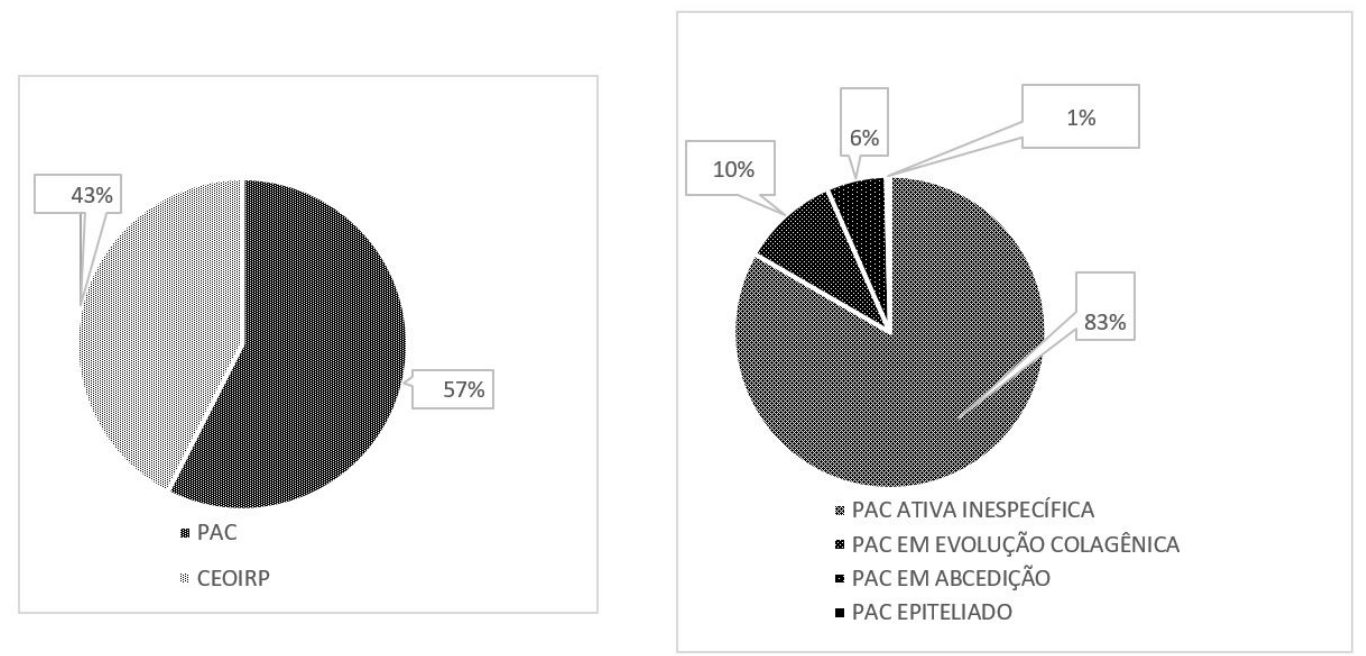

Gráfico 1: Quantidade das lesões diagnosticadas com laudo histopatológico. 
como periodontite apical crônica (PAC) e 7 deles como cisto inflamatório radicular (CEOIRP) (Gráfico 4), evidenciando, assim, a importância do exame histopatológico para a comprovação da lesão, tratamento e diagnóstico eficazes. Através desta pesquisa, comprova-se pelos seus resultados que os exames histopatológicos devem ser solicitados rotineiramente, diferenciando assim, conclusivamente, as lesões em questão, sendo indispensáveis, já que a diferenciação de PAC crônica e CEOIRP não se dá somente pelo exame clínico/radiográfico.

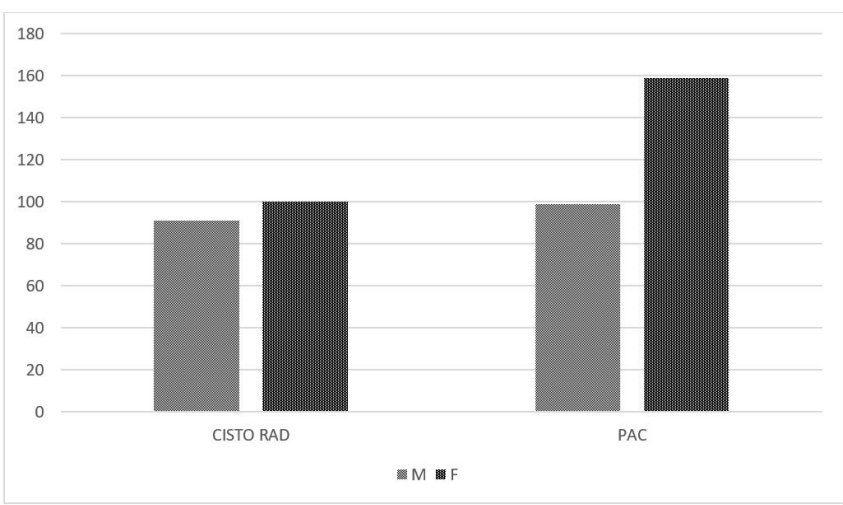

Gráfico 2: Distribuição das lesões quanto ao gênero
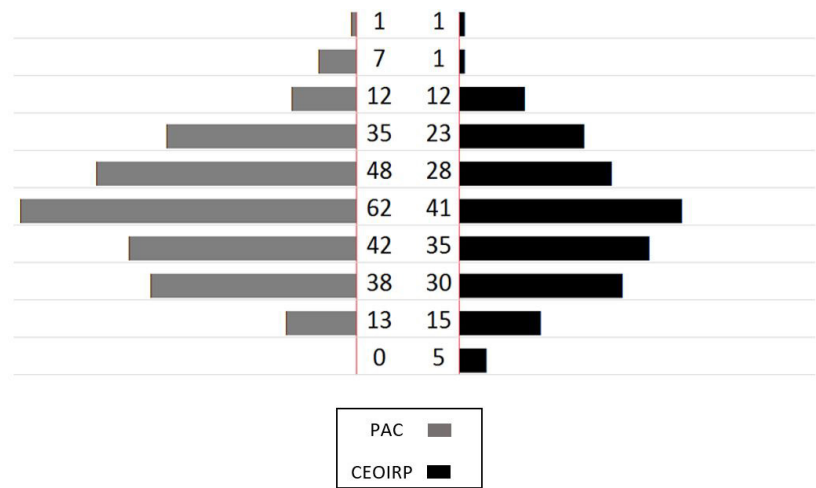

Gráfico 3: Faixas etárias de ambos os gêneros, PAC e CEOIRP, respectivamente.

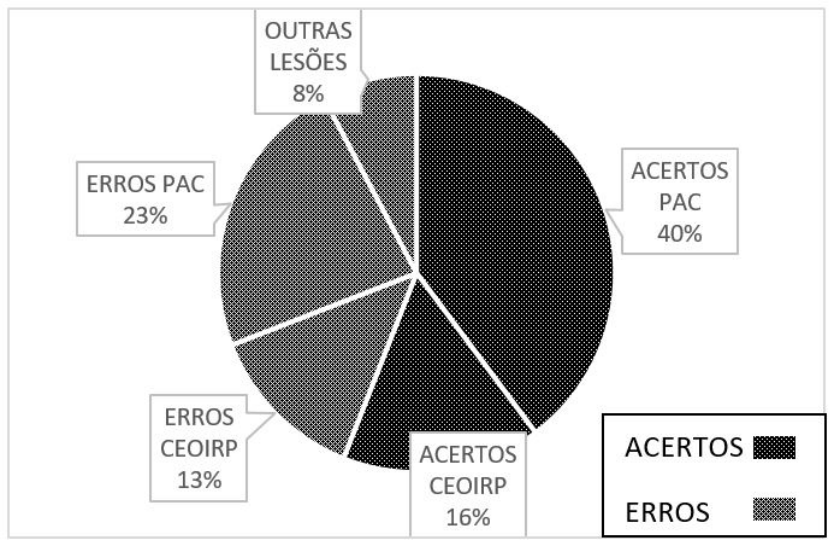

Gráfico 4: Porcentagem de concordância e discrepância referente ao diagnóstico clínico e histopatológico de PAC com suas variáveis e CEOIRP. 


\section{Conclusão}

De acordo com as condições experimentais, pelos resultados obtidos concluiu-se que:

- Há considerável discrepância entre diagnóstico clínico/radiográfico e diagnóstico histopatológico;

- Periodontite Apical Crônica Inespecífica foi o processo reacional predominante;

- O diagnóstico definido apenas pelos achados clínicos/radiográficos não é totalmente seguro na eficácia do tratamento;

- Um diagnóstico definitivo comprovado histopatologicamente confere ao cirurgião dentista maior segurança.

\section{Agradecimentos}

- Primeiramente à Deus pela força diária e por colocar anjos em forma de pessoas que sempre iluminam meus caminhos.

- Aos meus professores, orientadores e amigos Renata Hebling Marins e Raphael Carlos Comelli Lia, pois me nortearem neste trabalho.

- À minha família, na qual me embaso para sempre progredir.

- Ao namorado Felipe Camargo de Freitas e à amiga Tais Alves, sempre me incentivando e auxiliando durante todo o trabalho.

- Às colaboradoras Leninha e Terezinha, funcionárias do serviço de patologia, onde me receberam de braços abertos para todo o levantamento dos casos.

\section{Referências bibliográficas}

BAUMANN, L., \& ROSSMAN, S. R. (1956). Clinical roentgenologic, and histopathologic findings in teeth with apical radiolucent areas. Oral Surgery, Oral Medicine, and Oral Pathology, 9(12), 1330-1336. PMid:13378844. http://dx.doi. org/10.1016/0030-4220(56)90235-3

BHASKAR, S. N. (1966). Periapical lesions, types, incidence, and clinical features. Oral Surgery, Oral Medicine, and Oral Pathology, 21(5), 657-671. PMid:5218749. http://dx.doi.org/10.1016/00304220(66)90044-2

BLOCK, R. M., BUSHELL, A., RODRIGUES, H., \& LANGELAND, K. (1976). A histopathological, histobacteriologic and radiographic study of periapical endodontic surgical specimens. Oral Surgery, Oral Medicine, and Oral Pathology,
42(5), 656-678. PMid:1068421. http://dx.doi. org/10.1016/0030-4220(76)90217-6

FERREIRA, L. G. V., ROSIN, F. C. P., \& CORREA, L. (2016). Analysis of Interleukin $17 \mathrm{~A}$ in periapical abscess and granuloma lesions. Brazilian Oral Research, 30(1), http://dx.doi. org/10.1590/1807-3107BOR-2016.vol30.0034

GROSSMAN, L. I., \& ETHER, S. (1963). Estudo comparativo clínico e histológico-patológico de reações periapicais crônicas. Revista Brasileira de Odontologia, 22(124), 226-286. PMid:5338009.

LALONDE, E. R., \& LUEBKE, R. G. (1968). The frequency and distribution of periapical cysts and granulomas: an evaluation of 800 specimens. Oral Surgery, Oral Medicine, and Oral Pathology, 25(6), 861-868. PMid:5239741. http://dx.doi. org/10.1016/0030-4220(68)90163-1

LIA, R. C. C., GARCIA, J. M. Q., SOUSANETO, M. D., SAQUY, P. C., MARINS, R. H., \& ZUCOLLOTTO, W. G. (2004). Clinical, radiographic and histological evaluation of chronic periapical inflammatory lesions. Journal of Applied Oral Science, 12(2), 117-120. http:// dx.doi.org/10.1590/S1678-77572004000200007.

LINENBERG, W. B., WALDRON, C. A., \& DELAUNE JUNIOR, G. F. (1964). A clinical, roentgenographic, and histopathologic evaluation of periapical lesions. Oral Surgery, Oral Medicine, and Oral Pathology, 17(4), 467-472. PMid:14132867. http://dx.doi.org/10.1016/0030-4220(64)90040-4

MORSE, D. R., PATNIK, J. W., \& SCHACTERLE, G. R. (1973). Eletrophoretic differentiation of radicular cysts and granulomas. Oral Surgery, Oral Medicine, and Oral Pathology, 35(2), 249-264. PMid:4513070. http://dx.doi.org/10.1016/00304220(73)90292-2

MORTENSEN, H., WINTHER, J. E., \& BIRN, H. (1970). Periapical granulomas and cysts: an investigation of 1,600 cases. Scandinavian Journal of Dental Research, 78(3), 241-250. PMid:5273692.

NEVILLE, B. W., ALLEN, C. M., DAMM, D. D., \& BOUQUOT, J. E. (2004). Patologia: oral \& maxilofacial (2a ed.). Rio de Janeiro: Guanabara Koogan. 
NEVILLE, B. W., DAMM, D. D., ALLEN, C. M., \& BOUQUOT, J. E. (1998). Patologia oral e maxilofacial. Rio de Janeiro: Guanabara Koogan.

NEVILLE, B. W., DAMM, D. D., ALLEN, C. M., \& BOUQUOT, J. E. (2009). Patologia oral e maxilofacial (3. ed.). Rio de Janeiro: Elsevier.

PATTERSON, S. S., SHAFER, W. G., \& HEALEY, H. J. (1964). Periapical lesion associated with endodontically treated teeth. The Journal of the American Dental Association, 68(2), 191-194. PMid:14125029. http://dx.doi.org/10.14219/jada. archive.1964.0056

PRIEBE, W. A., LAZANSKY, J. P., \& WUEHRMANN, A. H. (1954). The value of the roentgenographic film in the differential diagnosis of periapical lesion. Oral Surgery, Oral Medicine, and Oral Pathology, 7(9), 979-983. PMid:13194304. http://dx.doi.org/10.1016/0030-4220(54)90297-2
REGEZI, J.A., SCIUBBA, J. J., \& POGREL, M. A. (2000). Atlas de patologia oral e maxilofacial. Rio de Janeiro: Guanabara Koogan.

ROCHA, M. M. N. P., MOREIRA, J. L. B., MENEZES, D. B., CUNHA, M. P. S. S., \& CARVALHO, C. B. M. (1998). Estudo bacteriológico de lesões periapicais. Revista de Odontologia da Universidade de Sao Paulo, 12(3), 215-223. http:// dx.doi.org/10.1590/S0103-06631998000300004

SHAFER, W. G., HINE, M. K. \& LEVY, B. M. (1987). Tratado de patologia bucal (4. ed.). Rio de Janeiro: Guanabara Koogan.

WAIS, F. T. (1958). Significance of findings following biopsy and histologic study of 100 periapical lesions. Oral Surgery, Oral Medicine, and Oral Pathology, 11(6), 650-653. PMid:13553318. http://dx.doi.org/10.1016/00304220(58)90012-4 